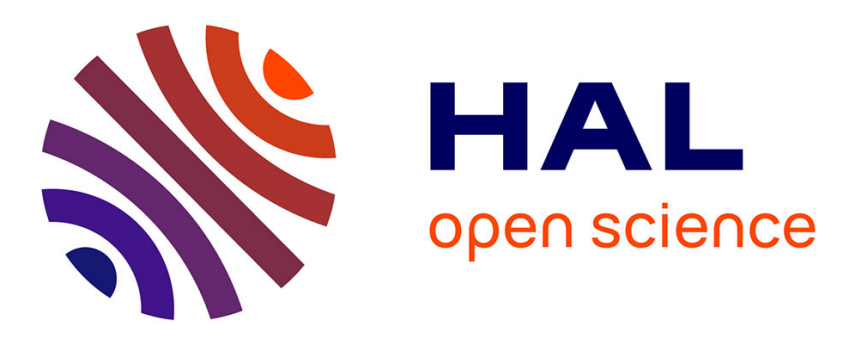

\title{
Secondary fast reconnecting instability in the sawtooth crash.
}

\author{
Daniele del Sarto, Maurizio Ottaviani
}

\section{To cite this version:}

Daniele del Sarto, Maurizio Ottaviani. Secondary fast reconnecting instability in the sawtooth crash.. Physics of Plasmas, 2017, 24, pp.12102 - 12102. 10.1063/1.4973328 . hal-01780899

\section{HAL Id: hal-01780899 \\ https://hal.science/hal-01780899}

Submitted on 16 May 2018

HAL is a multi-disciplinary open access archive for the deposit and dissemination of scientific research documents, whether they are published or not. The documents may come from teaching and research institutions in France or abroad, or from public or private research centers.
L'archive ouverte pluridisciplinaire HAL, est destinée au dépôt et à la diffusion de documents scientifiques de niveau recherche, publiés ou non, émanant des établissements d'enseignement et de recherche français ou étrangers, des laboratoires publics ou privés. 


\section{Secondary fast reconnecting instability in the sawtooth crash}

D. Del Sarto and M. Ottaviani

Citation: Phys. Plasmas 24, 012102 (2017); doi: 10.1063/1.4973328

View online: http://dx.doi.org/10.1063/1.4973328

View Table of Contents: http://aip.scitation.org/toc/php/24/1

Published by the American Institute of Physics 


\title{
Secondary fast reconnecting instability in the sawtooth crash
}

\author{
D. Del Sarto ${ }^{1, a)}$ and M. Ottaviani ${ }^{2, b)}$ \\ ${ }^{1}$ Institut Jean Lamour, UMR 7198 CNRS - Université de Lorraine, F-54506 Vandoeuvre-lés-Nancy, France \\ ${ }^{2}$ CEA, IRFM, F-13108 Saint-Paul-lez-Durance, France
}

(Received 9 March 2016; accepted 14 December 2016; published online 3 January 2017)

\begin{abstract}
In this work, we consider magnetic reconnection in thin current sheets with both resistive and electron inertia effects. When the current sheet is produced by a primary instability of the internal kink type, the analysis of secondary instabilities indicates that reconnection proceeds on a time scale much shorter than the primary instability characteristic time. In the case of a sawtooth crash, non-collisional physics becomes important above a value of the Lundquist number, which scales like $S \sim\left(R / d_{e}\right)^{12 / 5}$, in terms of the tokamak major radius $R$ and of the electron skin depth $d_{e}$. This value is commonly achieved in present day devices. As collisionality is further reduced, the characteristic rate increases, approaching Alfvénic values when the primary instability approaches the collisionless regime. Published by AIP Publishing. [http://dx.doi.org/10.1063/1.4973328]
\end{abstract}

\section{INTRODUCTION}

Current sheets are a common occurrence in laboratory and space plasmas. They appear as a local two-dimensional current concentration and they can be either the natural product of convective, often turbulent, plasma motion, or the result of a plasma instability entering its non-linear stage.

In this work, we study the instability of generic current sheets, addressing the question of whether reconnection can be fast enough to account for the observed rates of common events such as the sawtooth crash in a tokamak and possibly solar flares.

We consider a plasma characterized by a non-uniform average magnetic field $B_{0}$, varying on a characteristic scale length $L_{0}$. The structure of a current sheet is an almost planar current concentration of extension $L$ in two spatial dimensions and thickness $a \ll L$. Note that we keep $L$ and $L_{0}$ distinct although often in the literature $L \sim L_{0}$ or it is even assumed that $L=L_{0}$. By definition, the current density in the sheet would differ appreciably from the ambient current density. However one can argue, and it will be shown below, that such current concentration is unstable to fast growing perturbations. Therefore, the current density in the sheet $J_{C S}$ cannot exceed too much the ambient current density $J_{0}$, and therefore, one can reasonably assume $J_{c s} \sim J_{0} \sim B_{0} / L_{0}$.

Moreover, a current sheet would generally evolve in time. However, when studying its stability, it is convenient to assume it to be in a quasi-stationary state, if one is interested in instability growth rates much faster than the underlying evolution rate. As a consequence, a current sheet can be modeled as a planar system in local force balance. In the following, we further assume that the ambient magnetic field is almost constant and that the current density in the sheet is almost aligned to it.

The current in the sheet produces a magnetic field $B_{c s} \sim J_{c s} a$, which lays in the plane and it is transverse to the

${ }^{a)}$ Electronic mail: daniele.del-sarto@univ-lorraine.fr

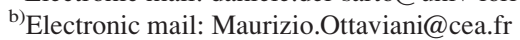

main magnetic field. From the previous estimate of $J_{c s}$, one concludes

$$
B_{c s} \sim B_{0} \frac{a}{L_{0}}
$$

This scaling is certainly pertinent when the current sheet is the result of a large $\Delta^{\prime}$ primary instability as in the case of the sawtooth phenomenon, which is discussed in detail in Sec. III. In this instance, scaling (1) marks the transition to the nonlinear phase if $a$ is the linear layer width. Therefore, in this context, we are assuming that the current sheet becomes unstable to a secondary instability at an early stage of the nonlinear evolution of the primary instability, a result we will find consistent with the estimates derived later in this work. Our logic will be outlined in a greater detail at the end of Sec. II.

Note also that, by assuming this scaling of $B_{c s}$ with the current sheet thickness, we depart fundamentally from previous works, ${ }^{1-11}$ where the scaling $B_{c s} \sim B_{0}$ is implicitly assumed. The implications of this different assumption are discussed more in detail in Sec. IV. Here, we anticipate that Pucci and Velli ${ }^{6}$ pointed out that by increasing the current sheet aspect ratio tearing mode theory predicts an increasing growth rate, reaching the Alfvénic limit at a characteristic aspect ratio that depends on the regime considered. ${ }^{6-11}$ The dependence of the growth rate on the aspect ratio is exploited in an essential way also in the present work, as discussed later.

Moreover, the presence of a strong guide field $B_{0}$ allows one to treat the problem in the framework of reduced MHD. In this respect, we depart from the work of Ref. 12, where the guide field is assumed zero.

In the following, we consider the resistive case, described by the model

$$
\begin{gathered}
\frac{1}{c_{A}^{2}}\left(\partial_{t} \nabla_{\perp}^{2} \hat{\boldsymbol{\phi}}+\hat{\boldsymbol{u}} \cdot \nabla \nabla_{\perp}^{2} \hat{\boldsymbol{\phi}}\right)=\nabla_{\|} \hat{\boldsymbol{J}}_{\|}, \\
\partial_{t} \hat{\psi}+\hat{\boldsymbol{u}} \cdot \nabla \hat{\psi}=-\hat{\eta} \hat{\boldsymbol{J}}_{\|},
\end{gathered}
$$


written in terms of the normalized stream function $\hat{\phi} \equiv c \phi / B_{0}$, where $\phi$ is the electrostatic potential, and of the normalized magnetic flux function $\hat{\psi} \equiv \psi / B_{0}$. Here, the current is given by $\hat{J}_{\|} \equiv-\nabla_{\perp}^{2} \hat{\psi}$, the velocity is $\hat{\boldsymbol{u}}=\boldsymbol{b}_{0} \times \nabla \hat{\phi}$, and the magnetic field is $\hat{\boldsymbol{B}}=\boldsymbol{b}_{0}-\boldsymbol{b}_{0} \times \nabla \hat{\psi}$, with $\boldsymbol{b}_{0}$, the unit vector of the local ambient magnetic field $\boldsymbol{B}_{0}$. Moreover, we have introduced the Alfvén velocity $c_{A}^{2} \equiv B_{0}^{2} /\left(4 \pi m_{i} n_{0}\right)$ and the magnetic diffusivity $\hat{\eta} \equiv c^{2} \eta /(4 \pi)$. The symbol $\|$ refers to the parallel component with respect to the total magnetic field, and $\perp$ refers to the components perpendicular to the local ambient field. Their exact expression depends on the context and they will be later specified in the case of slab and cylindrical geometry. Note that in Eqs. (2) and (3), lengths and times still appear in dimensional units.

In the following, we first derive the scaling of fast reconnection in a general large aspect ratio slab (Sec. II). Then, we apply the result to the sawtooth problem, by evaluating whether the sawtooth crash can result from the instability of the current sheet generated by a primary $m=1$ mode in a cylindrical tokamak (Sec. III). In particular, we compare our conclusions with the existing numerical results (Sec. III B), and we analyze the transition to the non-collisional regime. We finally point out the difference with other works on fast reconnection, and we outline possible future work (Sec. IV). The complementary material is given in the appendixes, with brief reviews of the notion of "fast" in reconnection literature (Appendix A), of numerical results and experimental observation of secondary reconnection (Appendix B), and of the asymptotic matching technique for stability calculations (Appendix C).

Before proceeding with Sec. II, we must point out that all the following calculations and results must be considered as an asymptotic analysis in terms of dimensionless parameters such as the normalized resistivity or its inverse (the Lundquist number), which will be considered arbitrary small (large) and the normalized electron skin depth. All quantities not depending on these parameters, such as certain geometrical factors like the tokamak aspect ratio, are assumed of order unity. Quantities of order unity will not be carried along in the calculations. Moreover, if a certain process occurs on a longer time scale (in the asymptotic sense) than another one, it will be considered as stationary for the purpose of computing the dominant behavior of the second one. Likewise, if a process has a characteristic length which is much smaller, in the asymptotic sense, than the scale length of the structure in which it is developed, it is reasonable to carry out a local analysis within a simplified geometry, much like what one would do in WKB theory.

Finally, we draw the attention of the reader to the fact that the secondary reconnecting instability we refer to in this paper is a standard tearing-type instability whose asymptotic scaling results to be compatible with experimentally observed disruptions in tokamaks because of geometrical rescaling arguments related to the current sheet aspect ratio: it is thus "fast" enough to possibly account for measurements but it is not necessarily Alfvénic, although we will show that its growth rate can attain the ideal regime as electron-inertia effects are taken into account. We refer the reader to Appendixes A and B for a summary on different examples of fast and nonlinear reconnection models frequently encountered in the literature.

\section{SCALING OF THE MAXIMUM RECONNECTION RATE}

Labeling with " $e q$ " the equilibrium quantities and leaving the perturbations unlabeled, we start by linearizing Eqs. (2) and (3) around an equilibrium with $\hat{\phi}_{e q}=0$ (no flow)

$$
\begin{gathered}
\frac{1}{c_{A}^{2}} \partial_{t} \nabla_{\perp}^{2} \hat{\phi}=\nabla_{\| e q} \hat{J}_{\|}+\nabla_{\|} \hat{J}_{\| e q}, \\
\partial_{t} \hat{\psi}+\nabla_{\| e q} \hat{\phi}=-\hat{\eta} \hat{J}_{\|},
\end{gathered}
$$

where $\nabla_{\|}=\hat{\boldsymbol{B}} \cdot \nabla$.

Adopting a local Cartesian coordinate system, we indicate with $x$ the coordinate perpendicular to the sheet, $y$ the direction of the transverse magnetic field $B_{c s}$, and $z$ the direction of the ambient field $B_{0}$. Moreover, for the sake of simplicity, one can assume a symmetric current distribution, such that $B_{c s}=0$ at $x=0$ and two dimensional perturbations such that $\partial_{z}=0$.

Using (1), in the neighborhood of $x=0$, the parallel gradient operator takes the form

$$
\nabla_{\| e q} \sim\left(\frac{B_{c s}^{\prime}(0)}{B_{0}}\right) k x \sim\left(\frac{k x}{L_{0}}\right) .
$$

The stability analysis of Eqs. (4) and (5) can be carried out with an asymptotic matching technique. A review of this known technique is given in Appendix C. In the following, instead, we directly work out the scaling of the growth rate $\gamma$ and of the inner reconnecting layer width $\delta$ in both the tearing (labeled as " $T$ ") and internal kink (labeled as " $K$ ") regimes. We recall that these regimes are identified by the conditions $\Delta^{\prime} \delta<1$ and $\Delta^{\prime} \delta>1$, respectively, where $\Delta^{\prime}$ is the usual tearing mode stability parameter. ${ }^{13}$ The scaling can be obtained, up to numerical constants, without carrying out the detailed asymptotic matching calculations, by taking $x \sim \delta$, so that $\nabla_{\| e q} \sim\left(k \delta / L_{0}\right)$. Moreover, using the fact that $k \ll \delta^{-1}, \partial_{x} \sim \delta^{-1}$ and the estimate $\hat{\phi}^{\prime \prime} \sim \hat{\phi} / \delta^{2}$, we obtain the following heuristic balance relations, respectively, from Eq. (4) (1.h.s. and first r.h.s. term) and from Eq. (5)

$$
\frac{1}{c_{A}^{2}} \frac{\gamma}{\delta^{2}} \hat{\phi} \sim \frac{k \delta}{L_{0}} \partial_{x}^{2} \hat{\psi}, \quad \gamma \hat{\psi} \sim \frac{k \delta}{L_{0}} \hat{\phi} \sim \hat{\eta} \partial_{x}^{2} \hat{\psi} .
$$

These can be now specialized by estimating the perturbed current density respectively as $\partial_{x}^{2} \hat{\psi} \sim \hat{\psi} / \delta_{K}^{2}$ for the internal-kink (large- $\Delta^{\prime}$ regime) and as $\partial_{x}^{2} \hat{\psi} \sim \hat{\psi} \Delta^{\prime} / \delta_{T}$ for the tearing mode (small- $\Delta^{\prime}$, constant- $\psi$ regime).

Introducing the macroscopic Alfvén time $\tau_{0} \equiv L_{0} / c_{A}$ and the Lundquist number referred to the macroscopic scales $S_{0} \equiv L_{0} c_{A} / \hat{\eta}$, the following scalings are obtained:

$$
\begin{aligned}
& \gamma_{T} \tau_{0} \sim S_{0}^{-\frac{3}{5}}\left(k L_{0}\right)^{\frac{2}{5}}\left(\Delta^{\prime} L_{0}\right)^{\frac{4}{5}}, \\
& \frac{\delta_{T}}{L_{0}} \sim S_{0}^{-\frac{2}{5}}\left(k L_{0}\right)^{-\frac{2}{5}}\left(\Delta^{\prime} L_{0}\right)^{\frac{1}{5}},
\end{aligned}
$$




$$
\begin{aligned}
& \gamma_{K} \tau_{0} \sim S_{0}^{-\frac{1}{3}}\left(k L_{0}\right)^{\frac{2}{3}} \\
& \frac{\delta_{K}}{L_{0}} \sim S_{0}^{-\frac{1}{3}}\left(k L_{0}\right)^{-\frac{1}{3}}
\end{aligned}
$$

Note that these scalings do not depend explicitly on the current sheet thickness $a$. However, in the tearing regime, they do depend implicitly on $a$ via $\Delta^{\prime}$, which also brings in an additional dependence on the wavenumber. If the aspect ratio $L / a$ is sufficiently large so that many wave-numbers are excited, a fastest growing mode exists ${ }^{13-16}$ at the wavenumber $k_{M}\left(S_{0}\right)$ corresponding to the transition between the tearing and the internal kink regimes. This transition occurs when $\Delta^{\prime} \delta \simeq 1$.

In order to see this, one has to specify the dependence of $\Delta^{\prime}$ on $a$ and $k$. Here, we adopt the expression obtained for the Harris ${ }^{17}$ pinch for small $k a$, that is, well above the tearing instability threshold

$$
\Delta^{\prime} \sim \frac{1}{k a^{2}}
$$

Note that the dependence $\sim 1 / k$ of $\Delta^{\prime}$ for small $k$ is fairly common and that, in any case, what follows can be easily generalized to a different ${ }^{10}$ power-law dependence.

Using Eq. (12) in Eqs. (9) and (11), we obtain

$$
\begin{aligned}
& k_{M} L_{0} \sim S_{0}^{-\frac{1}{4}}\left(\frac{L_{0}}{a}\right)^{\frac{3}{2}}, \\
& \gamma_{M} \tau_{0} \sim S_{0}^{-\frac{1}{2}}\left(\frac{L_{0}}{a}\right), \\
& \frac{\delta_{M}}{L_{0}} \sim S_{0}^{-\frac{1}{4}}\left(\frac{L_{0}}{a}\right)^{-\frac{1}{2}} .
\end{aligned}
$$

As indicated in the Introduction, the above results are of practical interest only if the underlying dynamics occurs at a rate lower than the estimate (14).

When the current sheet is the result of a large- $\Delta^{\prime}$ primary instability of a quiescent plasma, the time scale of its dynamics can be obtained from (10) by taking $k \sim L_{0}^{-1}$. This gives $\gamma_{\mathrm{I}} \tau_{0} \sim S_{0}^{-1 / 3}$. The corresponding layer width is an estimate of the current sheet width, $a \sim \delta_{\mathrm{I}} \sim L_{0} S_{0}^{-1 / 3}$. Moreover, as the system enters the nonlinear phase, the current density in the sheet is of the order of the equilibrium one and its magnetic field can be estimated as in Eq. (1). Henceforth, a full description of the magnetic structure would require a nonlinear treatment of the type carried out in Refs. 24 and 25. However, in the following, it is enough to assume that a current sheet centered on the (primary) $X$-point is produced, without further details on the precise form of the nonlinear structure. The existence of such a current sheet is indeed shown in Refs. 24 and 25, and observed in several numerical studies. In order to analyze the instabilities of such a structure, we further conjecture that in this early nonlinear phase the current sheet width still scales like the linear layer width of the primary instability. Any other hypothesis would require additional explanation. Finally, we assume that the (primary) nonlinear structure produced at the end of the primary instability phase evolves sufficiently slowly that it can be considered as stationary for the purpose of evaluating the secondary instability growth rate, a condition that can be verified a posteriori. As a reference evolution rate of this primary nonlinear structure, we can assume the linear growth rate of the primary instability. If anything, the evolution rate of the nonlinear structure issued from a resistive instability is usually found slower.

Using this information, one can estimate the maximum growth rate of the secondary instability from (14)

$$
\gamma_{M} \tau_{0} \sim S_{0}^{-\frac{1}{6}}
$$

which is clearly higher than the primary instability growth rate. One concludes that the current sheet produced when a high $\Delta^{\prime}$ primary instability enters the nonlinear phase becomes quickly unstable, as soon as the primary current sheet becomes sufficiently thin. An application of this mechanism to the sawtooth crash will be given in Sec. III.

More generally, current sheets occur as magnetic flux tubes are stretched and twisted by plasma advection. In an ideal plasma, this mechanism produces thinner and thinner current sheets. In a turbulent plasma with comparable kinetic and magnetic energy, the characteristic time is the Alfvén time $\tau_{0}$. Therefore, a current sheet will break up when its instability rate exceeds $\tau_{0}^{-1}$. From (14), one then deduces that the current sheet thickness must be of the order of

$$
\frac{a}{L_{0}} \sim S_{0}^{-\frac{1}{2}}
$$

Note that for $L_{0} \sim L$ this scaling is the same as the commonly quoted Sweet-Parker thickness. However, the analogy appears only superficial. This thickness has been obtained here as the necessary condition for the break-up of a sheet formed by Alfvénic motion rather than as the result of a steady reconnection process. Eq. (17) also represents the modified threshold aspect ratio for the trigger to the resistive "ideal" tearing mode in the quasi-ideal limit, as it is meant in Refs. 6 and 10, once the Alfvén time referred to the current sheet, $\tau_{c s}$, is rescaled to the macroscopic one according to $\tau_{c s}=\left(a B_{0}\right) /\left(L_{0} B_{c s}\right) \tau_{0}$, while assuming (1).

\section{SECONDARY FAST RECONNECTION AT THE TOKAMAK $n=m=1$ RESONANT SURFACE}

We now apply the above results to the $m=n=1$ internal kink (IK) instability in a large aspect ratio tokamak. The IK instability is considered an element of the sawtooth cycle, ${ }^{18}$ an almost periodic oscillation of plasma temperature characterized by a slow growth and a fast collapse. In the framework of resistive MHD, the IK instability is generally considered too slow to account for the observed fast sawtooth collapse, especially in weakly collisional plasmas, ${ }^{19}$ so that noncollisional effects are called in to explain observations. ${ }^{20-22}$

Here, we take a different approach. We consider a sufficiently resistive plasma, so that the IK can be treated with Eqs. (4) and (5), and we explore whether a secondary instability, possibly in the collisionless regime, can be fast enough to explain observations. 


\section{A. Primary internal-kink resistive mode}

For the sake of clarity, in this subsection, we review the key elements of the internal kink theory.

We consider a large aspect ratio tokamak in the periodic cylindrical approximation for which the poloidal equilibrium magnetic field $B_{\theta}^{0}$ is much smaller than the toroidal one, herewith identified by $B_{0}$. We adopt a set of cylindrical coordinates $(r, \theta, \varphi)$ for the radial, poloidal, and axial directions, respectively, with periodicity in $\theta$ and $\varphi$. We also recall the expression of the safety factor

$$
q(r) \equiv \frac{r B_{0}}{R B_{\theta}^{0}(r)},
$$

where $R$ is the major radius, the cylinder length being $2 \pi R$. Note that by allowing $q(r)$ to have both positive and negative signs, one can account for both a parallel and an anti-parallel equilibrium current. Looking for perturbations of the form $\hat{\psi}, \hat{J} \sim \cos (n \varphi+m \theta)$, and $\hat{\phi} \sim \sin (n \varphi+m \theta)$, where $m$ and $n$ can have both signs, replacing the time derivative $\partial_{t}$ with the growth rate $\gamma$, and using the corresponding cylindrical expression for the parallel gradient operator $\nabla_{\|}=(1 / R)$ $(\partial / \partial \varphi)+\hat{\boldsymbol{\varphi}} \times \nabla \hat{\psi} \cdot \nabla$, we can write the outer equations of the boundary layer problem as

$$
\begin{aligned}
0= & \left(n+\frac{m}{q(r)}\right)\left[\frac{m^{2}}{r^{2}} \hat{\psi}-\frac{1}{r} \frac{\partial}{\partial r}\left(r \frac{\partial}{\partial r} \hat{\psi}\right)\right] \\
& +\frac{m}{r} \hat{\psi} \frac{\partial}{\partial r}\left[\frac{1}{r} \frac{\partial}{\partial r}\left(\frac{r^{2}}{q(r)}\right)\right]
\end{aligned}
$$

and

$$
\gamma \hat{\psi}+\frac{1}{R}\left(n+\frac{m}{q(r)}\right) \hat{\phi}=0
$$

One can see that, for values of $m$ such that $m^{2}=1$, and assuming that a rational surface exists in the plasma at a position $r_{1}$ such that $\left|q\left(r_{1}\right)\right|=|1 / n|$ for some $n$, an exact solution of the above equations with vanishing boundary conditions exists. For both $m= \pm 1$, and up to an amplitude, this solution can be written as

$$
\hat{\psi}=r\left[n+\frac{m}{q(r)}\right], \quad \hat{\phi}=-\gamma r R
$$

inside the $r=r_{1}$ surface, and $\hat{\psi}=0, \hat{\phi}=0$ outside the $r=r_{1}$ surface. The discontinuity at $r=r_{1}$ together with the fact that $\hat{\psi}$ is zero at that position implies that $\Delta^{\prime}=\infty$ for these modes.

The case $|n|=1$ is of interest for the sawtooth problem in a tokamak.

In the neighborhood of the point $r_{1}$, which is contained in the inner reconnecting layer of width $\delta$, we can approximate

$$
\nabla_{\| e q} \simeq m \frac{\hat{s}_{1}}{R q_{1}} \frac{r_{1}-r}{r_{1}} \sim \frac{\delta}{L_{s} r_{1}}, \quad L_{s} \equiv\left|\frac{q_{1} R}{\hat{s}_{1}}\right|,
$$

where $L_{s}$ is the shear length in tokamak geometry, and $\hat{s}_{1}$ $=r_{1} q^{\prime}\left(r_{1}\right) / q_{1}$ is the magnetic shear at $r=r_{1}$, henceforth assumed as positive.
By carrying out the same balance as in the slab, large- $\Delta^{\prime}$ case, one obtains

$$
\gamma_{I} \tau_{0} \sim S_{R}^{-\frac{1}{3}}\left(\frac{r_{1}}{R \hat{s}_{1}}\right)^{-\frac{2}{3}}, \quad \frac{\delta_{I}}{R} \sim S_{R}^{-\frac{1}{3}}\left(\frac{r_{1}}{R \hat{s}_{1}}\right)^{-\frac{1}{3}},
$$

where in this instance the Alfvén time and the Lundquist number are defined using $R$ as a normalization length. The above results are identical to the slab case by taking $L_{0}=R / \hat{s_{1}}$, $k=r_{1}^{-1}$ and accounting for the change in the normalization length.

\section{B. Secondary instability and the sawtooth crash time scale}

As discussed in Sec. III A, at the end of the linear phase, and entering the nonlinear regime, a current sheet develops around the $X$-point, having the form of a helical ribbon of helicity $(1,1)$ and radius $r_{1}$. As a first approximation, we treat the problem as a planar sheet of width $\delta_{I}$, given by the inner layer width of the primary mode $m=n=1$. The neglect of the ribbon curvature, which is of the order of $1 / r_{1}$, is justified as in leading order WKB theory when the wavelength of interest is much smaller than the ribbon extension, a fact that can be verified a posteriori. Since at the end of the linear phase the perturbed current density is comparable to the equilibrium one, ${ }^{22,23}$ the magnitude of the transverse magnetic field in the current sheet, $B_{c s, \theta}^{I}$, can be estimated as

$$
\frac{B_{c s, \theta}^{I}}{B_{0}} \simeq \frac{x}{R}, \quad \text { for } x \leq \delta_{I} .
$$

As the IK instability enters the nonlinear phase, the extension of the sheet, $L$, would grow from a few times $\delta_{I}$, to a fraction of the circumference of radius $r_{1}$ (e.g., Waelbroeck estimates $^{25}$ it to be $\sim 2 \pi r_{1} / 3$ ), so we assume $\delta_{I}<L<r_{1}$. As the extension grows, higher and higher wave-numbers are progressively destabilized, with the smallest unstable wavevector given by $k_{\min } \equiv 2 \pi / L$.

We now adapt the results of Sec. II to the present context. In the following, we call $\delta_{I I}$ the layer width of the secondary instability and $\gamma_{I I}$ the corresponding growth rate. For the regime tearing of the secondary mode, we obtain

$$
\begin{aligned}
& \gamma_{I I}^{(T)} \tau_{0} \sim S_{R}^{-\frac{3}{5}}(k R)^{\frac{2}{5}}\left(\Delta^{\prime} R\right)^{\frac{4}{5}}, \\
& \frac{\delta_{I I}^{(T)}}{R} \sim S_{R}^{-\frac{2}{5}}(k R)^{-\frac{2}{5}}\left(\Delta^{\prime} R\right)^{\frac{1}{5}} .
\end{aligned}
$$

By estimating $\Delta^{\prime}$ as in (12), with $a$ replaced by the IK layer width $\delta_{I}$, one obtains

$$
\begin{gathered}
\gamma_{I I}^{(T)} \tau_{0} \sim S_{R}^{-\frac{1}{15}}(k R)^{-\frac{2}{5}}\left(\frac{r_{1}}{\hat{s_{1} R}}\right)^{-\frac{8}{15}}, \\
\frac{\delta_{I I}^{(T)}}{R} \sim S_{R}^{-\frac{4}{15}}(k R)^{-\frac{3}{5}}\left(\frac{r_{1}}{\hat{s_{1} R}}\right)^{-\frac{2}{15}} .
\end{gathered}
$$

The fastest growing mode has a wavenumber 


$$
k_{M}^{(I I)} R \sim S_{R}^{\frac{1}{4}}\left(\frac{r_{1}}{\hat{s_{1}} R}\right)^{-\frac{1}{2}}
$$

which belongs to the available range as $L$ becomes comparable to $r_{1}$.

The corresponding maximum growth rate is

$$
\gamma_{I I}^{(M)} \tau_{0} \sim S_{R}^{-\frac{1}{6}}\left(\frac{r_{1}}{\hat{s_{1} R}}\right)^{-\frac{1}{3}} .
$$

By integrating the reduced-MHD (RMHD) equations in cylindrical geometry, Yu et al. ${ }^{26}$ have found that the current layer generated by the nonlinear growth of a primary $m / n=1 / 1$ magnetic island becomes strongly unstable to secondary tearing modes.

According to our calculation (30), in the secondary island regime, a weak positive dependence of the reconnection time on $S_{R}, \sim S_{R}^{1 / 6}$, is expected. This appears consistent with the dependence shown in Fig. 5 of $\mathrm{Yu}$ et al. ${ }^{26}$ (black bullets).

In many middle-size tokamaks, the rate given by Eq. (30) is probably fast enough to account for the observed evolution of the sawtooth crash. To assess the validity of the resistive model, one has to compare the predicted rate with the electron collision rate $\nu_{e}$. Using the fact that $\hat{\eta} \sim \nu_{e} d_{e}^{2}$, where $d_{e}$ is the electron skin depth, and ignoring geometrical factors, one can consider two cases.

For the validity of Eq. (23) (primary instability), one requires $\gamma_{I}<\nu_{e}$, which can be recast as

$$
\tau_{0} \nu_{e}>\frac{d_{e}}{R}
$$

On the other hand, for the validity of Eq. (30) (secondary instability), one requires $\gamma_{I I}<\nu_{e}$, which gives the more restrictive condition

$$
\tau_{0} \nu_{e}>\left(\frac{d_{e}}{R}\right)^{\frac{2}{5}}
$$

In the intermediate regime

$$
\frac{d_{e}}{R}<\tau_{0} \nu_{e}<\left(\frac{d_{e}}{R}\right)^{\frac{2}{5}}
$$

the primary instability can be treated with resistive MHD, while a non-collisional model is required to treat the secondary instability correctly.

Finally, when condition (31) is also violated, the growth rate of the primary instability also depends on noncollisional physics. $^{21-23}$

The three collisionality regimes are summarized in Fig. 1. Here, the green line indicates the normalised collision frequency as a function of the Lundquist number. The red lines represent the growth rates in the resistive regime. When they are below the green line, the growth rate is smaller than the collision frequency and the resistive theory holds. One can see from the figure that for $S<\left(L_{0} / d_{e}\right)^{12 / 5}$ both the primary and the secondary instability can be treated with

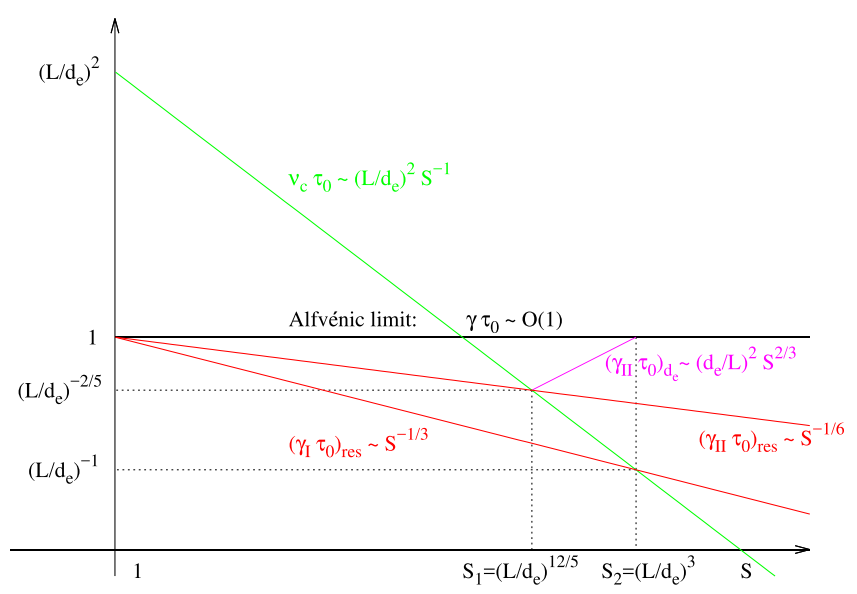

FIG. 1. Sketch in log-log scale of the maximum growth rate of the secondary instability as a function of the Lundquist number in the collisional (red) and in the non-collisional (mauve) regime. Also shown are the growth rate of the primary instability (also red) and the collision frequency (green). Here, $L_{0} \sim L$ has been specified.

resistive MHD. On the other hand, when $S>\left(L_{0} / d_{e}\right)^{3}$, none of the growth rates is less than the collision frequency, and noncollisional effects must be taken into account. Finally, in the intermediate regime $\left(L_{0} / d_{e}\right)^{12 / 5}<S<\left(L_{0} / d_{e}\right)^{3}$, the primary instability is resistive, but noncollisional effects are necessary to treat the secondary instability, whose growth rate is given by the mauve line.

The effect of non-collisional physics can be evaluated by considering the model with electron inertia ${ }^{22}$ and adapting the estimates of Sec. II (cf. Eqs. (C10)-(C13) in Appendix C). Then, by using again $\Delta^{\prime} \sim 1 / k a^{2}$, and $R \sim L_{0}$, Eqs. (8)-(11) are replaced by

$$
\begin{gathered}
\gamma_{T} \tau_{0} \sim\left(\frac{d_{e}^{3} L_{0}}{a^{4}}\right)\left(k L_{0}\right)^{-1}, \\
\frac{\delta_{T}}{L_{0}} \sim\left(\frac{d_{e}^{2}}{a^{2}}\right)\left(k L_{0}\right)^{-1}, \\
\gamma_{K} \tau_{0} \sim\left(\frac{d_{e}}{L_{0}}\right)\left(k L_{0}\right), \\
\frac{\delta_{K}}{L_{0}} \sim \frac{d_{e}}{L_{0}} .
\end{gathered}
$$

The growth rate and the layer width as a function of the wavenumber are sketched in the log-log plot in Fig. 2 for the collisional (Eqs. (8)-(11) with $\Delta^{\prime}$ given by (12)) and noncollisional regime (Eqs. (34)-(37)).

In the intermediate regime, the situation with the two (collisional and non-collisional) estimates is summarized in Fig. 3.

One can see that, apart from a range at the largest wavenumbers, the growth rate of the secondary instability is determined by non-collisional physics. In particular, the peak growth rate, occurring at the transition between (34) and (36), scales like

$$
\gamma_{M} \tau_{0} \sim \frac{d_{e}^{2}}{a^{2}}
$$




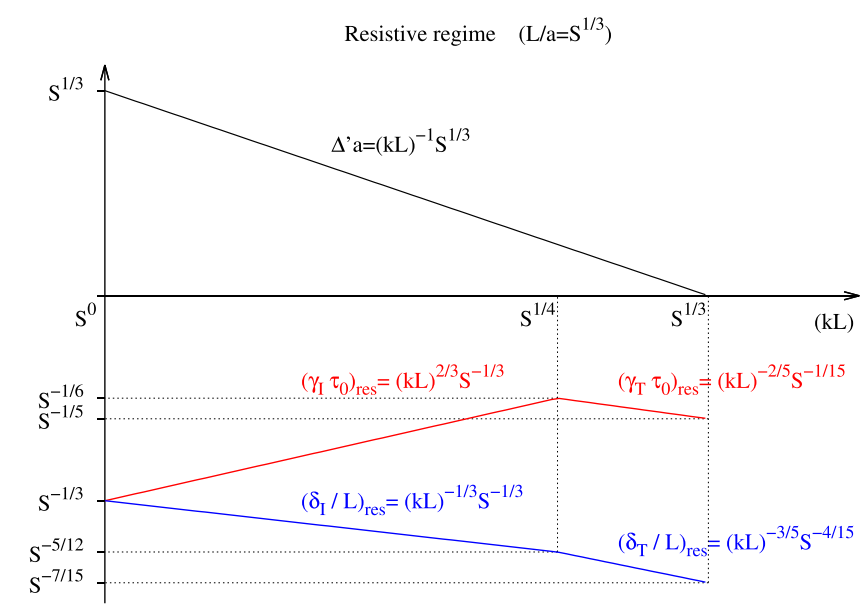

(a)

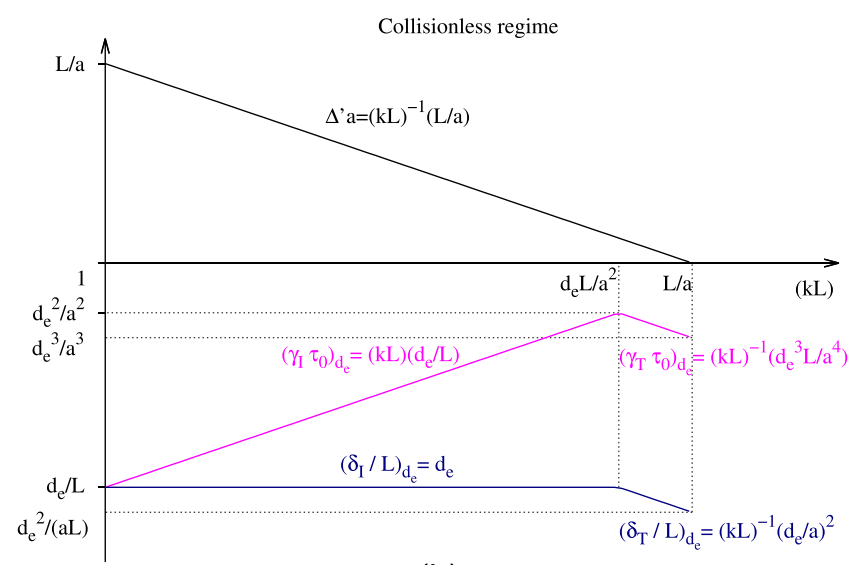

(b)

FIG. 2. Growth rate, layer width, and $\Delta^{\prime}$ as a function of the wavenumber for the collisional (a) and the non-collisional (b) theory. $L_{0} \sim L$ has been specified.

As one approaches the right boundary of the intermediate regime, the thickness of the primary layer decreases, we recall, as $\sim L_{0} S^{-1 / 3}$. Thus, the maximum growth rate of the secondary instability grows again as $\sim S^{2 / 3}$. This behavior is also shown in Fig. 1 (mauve line $\left(\gamma_{I I} \tau_{0}\right)_{d_{e}}$ ). At the right boundary of this regime, $S \rightarrow\left(L_{0} / d_{e}\right)^{3}$ and $a \rightarrow d_{e}$. Then, the current sheet produced by the primary instability approaches the electron skin depth, while the maximum growth rate of the secondary instability approaches Alfvénic values.

The growth rate (38) is fast enough to account for the observed sawtooth crash time in a machine like JET. ${ }^{19}$ These observations call for a non-collisional theory since the observed rates are faster than the collision frequency. In this respect, referring again to Fig. 1, we notice that our approach brings the boundary, beyond which the collisionless effects matter, down to $S \sim\left(R / d_{e}\right)^{12 / 5}$. This value of the Lundquist number is much more easily achieved in large tokamaks than $S \sim\left(R / d_{e}\right)^{3}$ at which the primary instability enters the collisionless regime (upper boundary of the intermediate regime). One can also remark that the reconnection rate has a minimum that scales like $\sim\left(d_{e} / L_{0}\right)^{2 / 5}$, in Alfvén units, in all collisionality regimes.

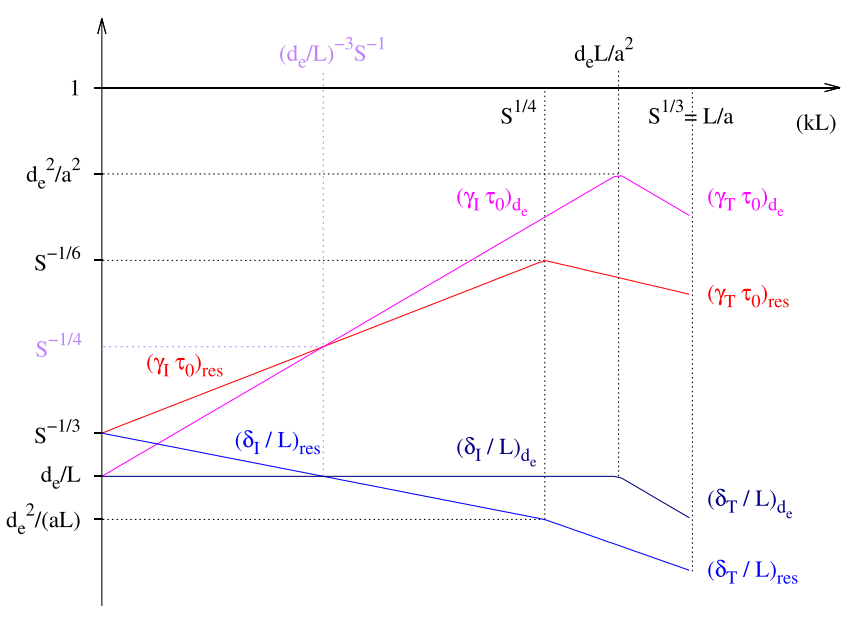

FIG. 3. The scalings of the resistive (label "res") and of the purely inertiadriven (label " $d_{e}$ ") regimes at $L=L_{0}$ and for $\left(L / d_{e}\right)^{12 / 5}<S<\left(L / d_{e}\right)^{3}$ are, respectively, given by (10) and (36) for the primary growth rates $\left(\gamma_{I} \tau_{0}\right)$ and by (8) and (34) for the growth rates of the secondary tearing modes $\left(\gamma_{T} \tau_{0}\right)$. Similarly, (11), (37) and (9), (35) respectively give the inner layer widths $\delta_{I} / L$ and $\delta_{T} / L$ of the secondary reconnecting modes. $L_{0} \sim L$ has been specified.

\section{DISCUSSION AND CONCLUSIONS}

The results of Yu et al. ${ }^{26}$ have also been interpreted ${ }^{27}$ in terms of the plasmoid instability. ${ }^{3}$ However, it appears that the literature on the plasmoid instability does not take into account the rescaling of the current sheet magnetic field with respect to its macroscopic, reference value (Eq. (1)) and, under these hypotheses, the assumption that the current layer aspect ratio scales $\grave{a}$-la Sweet-Parker, which is at the basis of the plasmoid instability scaling, ${ }^{3,6,10,34}$ becomes questionable. ${ }^{6}$ We recall that the rescaling (1) is necessary to estimate correctly the size of the current sheet magnetic field at the end of the linear phase of a primary instability such as the $m=n=1$ mode in the sawtooth phenomenon.

By ignoring such rescaling, the secondary instability is faster, such that the maximum growth rate given in Eq. (14) would be replaced by ${ }^{6}$

$$
\gamma_{M} \tau_{0} \sim S_{0}^{-\frac{1}{2}}\left(\frac{L_{0}}{a}\right)^{\frac{3}{2}}
$$

By assuming that the width of the current sheet resulting from the primary instability scales like $\sim S_{R}^{-1 / 3}$, as in this work, together with estimate (39), one would conclude that the maximum growth rate scales like the ideal tearing growth rate $\gamma_{I I} \tau_{0} \sim O(1)$, independent of resistivity.

These assumptions are at the root of the work of Pucci and Velli as a criterion for an ideal tearing reconnection rate in a purely resistive model. ${ }^{6,8,9}$

If on the other hand one assumed a narrower current layer scaling $\grave{a}$-la Sweet-Parker, $a \sim L_{0} S_{0}^{-1 / 2}$, and at the same time one ignored the rescaling (Eq. (1)), thereby using again estimate (39), one would end up with a faster-than Alfvénic estimate of the maximum growth rate $\sim S_{0}^{1 / 4}$, which gives, for $L_{0}=L$, the plasmoid instability scaling. ${ }^{28}$

Therefore, in the scenario where the current sheet is produced by a primary instability, as in the sawtooth case, we consider the results based on the plasmoid instability unlikely, since we have shown that the current sheet becomes sufficiently 
unstable to secondary sub-Alfvénic modes at an earlier stage in its development and it would therefore likely break up before becoming sharper, in current concentration, or thinner, in width, than the estimates of Eqs. (1) and (11), respectively.

In summary, in this work, we have shown that a current sheet generated by a primary instability, such as the internal kink mode, becomes sufficiently unstable at an early stage in its nonlinear development.

When applied to the context of the sawtooth phenomenon, the analysis of the secondary instability reveals that the sawtooth crash due to reconnection proceeds at a faster rate than previous estimates based on the primary $m=n=1$ internal kink mode. ${ }^{22}$ In the case of a purely resistive model, the reconnection rate depends weakly on the Lundquist number, $\sim S^{-1 / 6}$, and it appears in agreement with the results of numerical simulations at a large Lundquist number. ${ }^{26}$ Also, the value of the Lundquist number at which collisionless effects become important is found to be substantially lower than the estimate based on the primary instability only. This broadens the range of tokamaks to which collisionless effects should be taken into account in the analysis of the sawtooth phenomenon. Finally, near-Alfvénic reconnection rates can be achieved by secondary instabilities when collisionless effects become important also for the primary instability.

The scope of this work is to outline a possible scenario for fast reconnection, and in this respect, scaling estimates are useful, and sufficient for a first investigation.

Detailed stability analysis of current sheets, taking into account the actual geometry, such as the $m=1$ ribbon, is a possibility to obtain more precise predictions about the maximum growth rate, the associated wavenumber, and the instability threshold. This might lead to an explanation of the Lundquist number threshold observed in numerical simulations $^{26}$ and of the number of observed secondary islands.

The effect of flows also merits an investigation. In this respect, one notes that flows are small near the primary instability $X$-point, which is a stagnation point, but may be significant far from it, potentially leading to stabilizing effects on the tearing mode $e^{9,29-31}$ and/or to additional instabilities such as KelvinHelmholtz's. ${ }^{32-34}$ In particular, according to Bulanov et al. ${ }^{30}$ and Syrovatskii, ${ }^{31}$ flow stabilization would occur if its derivative along the reconnection line would roughly exceed the instability growth rate. Our estimates indicate that the former scales like $S_{0}^{-1 / 3}$ at the end of the linear phase of the primary instability, whereas the latter scales, as we have seen, at least as $S_{0}^{-1 / 6}$. Thus, the criterion is not met and the flow can be neglected.

Finally, the stability analysis carried out in this work is based on a magnetic configuration with the current aligned to a main magnetic field. Relaxing this hypothesis and allowing for pressure gradients would open further possibilities.

\section{ACKNOWLEDGMENTS}

The authors wish to thank Q. Yu for discussions about his work, A. Tenerani for interesting discussions and for details about Ref. 9, and L. Comisso for having brought attention to Ref. 28. Discussions with F. Pucci and M. Velli on the ideal tearing are also gratefully acknowledged.

\section{APPENDIX A: NOTIONS OF FAST RECONNECTION}

For the sake of clarity, let us briefly review the use of the adjective "fast" in the context of the reconnection literature, since its meaning has been changing over the years and in different contexts.

Until about the 1990s, especially in tokamak-related literature, "fast" refers to a reconnection rate that is faster than what is expected by the resistive tearing $\operatorname{mode}^{13}$ and SweetParker $^{35,36}$ scalings, which are too slow to account for experimental evidence. ${ }^{19}$ From the theoretical viewpoint, "fast" refers to effects beyond resistive MHD, such as electron inertia, ${ }^{20-22}$ that allow a finite reconnection rate even when the resistivity is negligibly small. This is the meaning adopted in this work. We also note that the word "collisionless" has been frequently used for this latter purpose, too, but while in origin it meant a reconnection triggered by (electron) inertia effects replacing resistivity (see, e.g., Refs. 21, 22, 37, and 58), it has later been used to generically underline the role of collisionless effects, in particular, the Hall-term, in enhancing resistivity-driven reconnection rates (see, e.g., Refs. 39-41). This change of terminology relates to that for the notion of fast reconnection.

In more recent literature, the word "fast" seems to have taken the more restrictive meaning of reconnection at ideal time scales (Alfvénic or even super-Alfvénic), essentially after the whistler-mediated (or Hall-mediated) reconnection model, ${ }^{38,39}$ displaying a reconnection rate weakly dependent on the non-ideal parameters, has been recognized as the paradigm for magnetospheric reconnection. ${ }^{40-42}$

We recall however that the Hall-term is negligible in the strong guide field, slab geometry approximation pertinent to tokamaks and considered here, where it is related at most to ion-sound Larmor radius effects (see, e.g., Refs. 10 and 43-45), well known to increase the "cold" resistive reconnection rate ${ }^{21}$ but without allowing the transition to the whistler-mediated regime.

Starting from the 2000s, different models for this more recent meaning of "fast" reconnection have been then considered as relevant to secondary reconnection events: the plasmoid-induced, fractal reconnection model, ${ }^{1}$ which the more recent plasmoid instability ${ }^{3}$ is reminiscent of, and then the "ideal" tearing model. ${ }^{6}$ As discussed in the recent review 11, however, all of them essentially consist of tearing-type instabilities that generate "plasmoids," i.e., magnetic islands, with different growth rate scalings that depend on the scalings of the current sheet aspect ratio. In this regard, it is in Ref. 6 that a distinction was first made between reconnecting modes classified as "slow" (i.e., ideally stable), "fast" (i.e., (quasi-)ideally unstable), and "violently unstable" (i.e., with diverging growth rates while approaching the ideal limit).

\section{APPENDIX B: BRIEF REVIEW OF NUMERICAL RESULTS AND EXPERIMENTAL OBSERVATIONS OF SECONDARY RECONNECTING INSTABILITIES}

As the notion of secondary reconnection requires the primary reconnection event to be treated as relatively "stationary" with respect to the secondary growth rate $\left(\tau_{r e c, I}^{-1} \ll \gamma_{I I}\right)$, it is natural that such an occurrence has been 
first evidenced as generation of "plasmoids" on almost stationary current-sheets that were reconnecting à la SweetParker ${ }^{48,49}$ or developing between two coalescing magnetic islands $^{50}$ (see also Ref. 14). For long time, secondary reconnection has been thus refereed to primary Sweet-Parker-like reconnection rates. It is in this sense, for example, that radio pulsations measured in solar flares have been related to the dynamics of magnetic islands secondarily generated on a quasi-stationary reconnecting current sheet. 51

On the other hand, it was known from the theoretical works by Syrovatskii ${ }^{52}$ (see also Ref. 31 and references therein) that current sheets could form from the collapse of an $X$-point into the so-called two- $Y$-point configuration. Besides the coalescence instability problem, ${ }^{46,47}$ where the collapse of the $X$-point in-between two coalescing flux ropes is driven by the mutual attraction of the two current patches, the double- $Y$-point configuration could naturally evolve from $X$-points generated by primary tearing modes. Evidence of this was provided, for example, in the numerical studies of the nonlinear tearing mode ${ }^{22,23,58}$ (see, in particular, Fig. 13 of Ref. 23), whose dynamics had been already shown by analysis to imply the formation of a current sheet, ${ }^{24}$ or in studies dedicated to the investigation of the $X$-point collapse. ${ }^{53}$ The computational resources available a few years later allowed us to evidence the occurrence of secondary instabilities: fluid-type instabilities were shown to affect the current sheet evolution in a primary collisionless internal kink mode ${ }^{32}$ or in large wave-length EMHD reconnection, ${ }^{54}$ in agreement ${ }^{33}$ with the importance of the electron KelvinHelmholtz mode for the instability of thin current layers in collisionless regimes. ${ }^{55}$ Then, in resistive regimes, also secondary reconnecting instabilities were shown to occur: it is essentially after Refs. 2, 56, and 57, which showed an increase of the reconnection rate due to the formation of plasmoids in the current sheet generated by the collapse of magnetic island $X$-points, that the secondary reconnection rate started being compared to primary tearing-type modes, too. It is also worth noticing that, looking retrospectively, Aydemir's early numerical investigation of the internal kink in cylindrical geometry ${ }^{58}$ contains elements of secondary instabilities in a collision-less regime, although at the time different interpretations were sought for the observed reconnection rate increase.

While this seems to summarize the main stream of plasma literature on secondary reconnection until about the first half decade of the 2000s, we note that more specifically astrophysic-oriented works had already evidenced ${ }^{1,59}$ how fast, secondary tearing modes could develop on the current sheet generated during the nonlinear evolution of a primary tearing (in turn induced, in the case studied in Ref. 59, by a shock-wave trespassing a current sheet). Some specific words are due, in particular, to the pioneering works by Shibata and Tanuma, who, between the end of 1990s and begin of 2000s, devised the plasmoid-induced reconnection $^{1,60}$ and the fractal reconnection ${ }^{1}$ models to account for nonlinear fast reconnection processes: the former highlights the role of plasmoids, generated by primary reconnection events and ejected along the current sheet, in enhancing the inflow and thus the reconnection rate at $X$-points because of mass conservation between the inflow and outflow. The latter considers how this may contribute to a cascade, fractal-like process, in which a sequence of increasingly faster tearing modes develop each on the shoulder of the previous one, after a sufficiently large aspect ratio current sheet has formed from the $X$ point collapse of each tearing. A re-scaling argument analogous to the one discussed here and the assumption of a Sweet-Parker scaling for each current sheet aspect ratio are two ingredients of this model, in which the growth rate of a secondary tearing was probably first compared to a previous one.

The interpretation of the current sheet generated by the $X$-point collapse as a Sweet-Parker state, also assumed in Refs. 2 and 53, led a few years later to the formalization of the plasmoid instability, ${ }^{3}$ which thereafter became indicative of a specific positive scaling of $\gamma$ with $S$ in the strong guide field, slab geometry approximation (for a brief review of the previous usage of the term "plasmoid" in reconnection, especially in astrophysics, see, e.g., Ref. 62). As Tajima and Shibata earlier pointed out, ${ }^{28}$ however, $\gamma \tau_{0} \sim S_{0}^{1 / 4}$ and $k_{M} L \sim S_{0}^{3 / 8}$ are the scalings of the most unstable tearing mode in a Sweet-Parker current sheet when $L_{0}=L$, fact which highlights the tearing nature of the plasmoid instability. After Ref. 3, many numerical results, starting from Refs. 16 and 61 , have been interpreted in terms of the plasmoid instability scaling, including ${ }^{27}$ the recent results by $Y u$ and co-workers ${ }^{26}$ on the nonlinear evolution of an internal kink mode during the sawtooth cycle in a purely resistive regime.

Alternative interpretations have been nevertheless provided for the almost ideal reconnection rate $\tau_{\text {rec }}^{-1} \tau_{0} \sim S_{0}^{\alpha}$ with $\alpha \simeq 0$, often numerically measured in correspondence of the generation of secondary islands (see, e.g., Refs. 8-11, 27, and 63-70), which are based on 3D effects, on reconnection mechanisms of kinetic nature and/or on two-fluid effects, or on the linear or quasi-linear analysis of secondary reconnecting modes. Some of the latter have been sought following the recent criticism to the Sweet-Parker scaling assumption-see in this regard the "ideal"-tearing based models, ${ }^{6-11}$ which first have raised it in evidencing a threshold aspect ratio of the current sheet to ideal reconnection rates that is smaller than $L / a \sim S^{1 / 2}$, and Refs. 69 and 70 .

In particular, numerical evidence of two "ideal" tearing modes, developing in sequence, one secondary to the other, has been first provided in Ref. 8. In Ref. 9, this mechanism has been shown to iterate in a cascade process akin to the fractal reconnection model (see also Ref. 11 for a comparison of the two), in which the stabilizing role of flows becomes progressively more important. The assumption $B_{c s} \sim B_{0}$, which implies $J_{c s} \gg J_{0}$ when $L_{0} / a \gg 1$, seems to be verified ${ }^{9,11}$ in these models, of which it is a key ingredient. As we have already pointed out, this is not the case for the current sheet generated by primary "non-ideal" resistive tearing modes, for which $J_{C S}$ is disrupted by secondary instabilities before it overtakes too much the macroscopic current sheet $J_{0}$. Therefore, the estimation provided in Ref. 10 to account for the possible onset of a secondary "ideal" regime by starting from a primary "non-ideal" tearing mode or for the secondary reconnection rate increase, sometimes evidenced in simulations as the explosive reconnection regime, ${ }^{64,66}$ should be corrected in the light of Eqs. (8)-(11), (C10)-(C13). 
Regardless of the assumptions implying different scaling estimations, the reconnecting instabilities leading to secondary magnetic island formation can be essentially treated as tearing-type modes (see, e.g., Ref. 11), as long as a WKBtype approach makes it possible to apply a linear instability analysis on a relatively slowly evolving current sheet.

Secondary magnetic islands generated next to $X$-points of primary reconnecting modes have been observed also in numerical simulations of turbulent reconnection ${ }^{71}$ and have been evidenced by in situ satellite measurements in the magnetosphere. ${ }^{72,73}$ The formation of secondary islands, due to the nonlinear reconnection of magnetic lines on different rational surfaces, has also been pointed out in early numerical studies of the double-tearing mode in the collision-less regime, ${ }^{74}$ and, more recently, the reconnection rate has been observed to abruptly increase during the nonlinear stage of a primary double-tearing mode up to almost ideal scalings ${ }^{75-77}$ In Ref. 77, in particular, a geometrical threshold to the onset of secondary linear modes, growing almost independent of $S$, has been identified in terms of the $2 \mathrm{D}$ structure that the primary magnetic islands achieve during the nonlinear evolution of the initial double-tearing.

A cascade of reconnecting instabilities from an initial reconnecting mode at macroscopic scales down to reconnection below the ion-skin depth scale has been evidenced by Moser and Bellan in laboratory experiments of a magnetized current carrying plasma jet. ${ }^{81}$ More recently, plasmoid generation due to a secondary resistive tearing on a current sheet generated by driven, quasi-steady reconnection, has been measured in experiments with the MRX device. ${ }^{78}$ Thanks to the recent improvements of the diagnostic systems, and, in particular, of the soft $\mathrm{X}$-ray and electron cyclotron emission imaging, also the growth of secondary reconnecting instabilities during the sawtooth phenomenon in tokamaks (itself known since the first observations reported in Ref. 18) has been experimentally measured. Examples are provided by Ref. 79 for the HT-7 device and by Ref. 80 for the ASDEX-Upgrade tokamak.

\section{APPENDIX C: SOME ELEMENTS OF THE STABILITY ANALYSIS}

In this appendix, we review the stability analysis of Eqs. (4) and (5) with the asymptotic matching technique. We give only the key elements as the procedure is already known. In the region around the boundary layer at $x=0$, such that $x \ll L_{0}$, called the inner region, we can approximate the parallel derivative operator as in Eq. (6)

$$
\nabla_{\| e q} \approx\left(\frac{B_{c s}^{\prime}(0)}{B_{0}}\right) x \partial_{y}=\left(\frac{x}{L_{0}}\right) \partial_{y}
$$

where the shear length $L_{s} \equiv B_{0} / B_{c s}^{\prime}(0)$ can be identified with the generic macroscopic scale length $L_{0}$ for our purposes.

Consider then perturbations of the form $\hat{\psi} \sim e^{\gamma t} \cos (k y)$ and $\hat{\phi} \sim e^{\gamma t} \sin (k y)$. Assume also a strong radial variation across the boundary layer, such that in the inner region one can take $\partial_{x} \gg k$, and keep only the leading terms. Then, Eqs. (4) and (5) become

$$
\begin{gathered}
\frac{1}{c_{A}^{2}} \gamma \hat{\phi}^{\prime \prime}=k\left(\frac{x}{L_{0}}\right) \hat{\psi}^{\prime \prime}, \\
\gamma\left(\hat{\psi}-d_{e}^{2} \hat{\psi}^{\prime \prime}\right)+k\left(\frac{x}{L_{0}}\right) \hat{\phi}=\hat{\eta} \hat{\psi}^{\prime \prime} .
\end{gathered}
$$

Here, the electron inertia term proportional to the square of the electron skin depth $d_{e}$ has been introduced as a first step to include noncollisional effects.

By first defining a length $\delta_{e}$ such that $\delta_{e}^{2} \equiv d_{e}^{2}+\hat{\eta} / \gamma$, and then by introducing the parameter $Q \equiv \gamma L_{0} /\left(k C_{A} \delta_{e}\right)$, and the length $\delta_{L} \equiv Q^{1 / 2} \delta_{e}$, one can recast Eqs. (C2) and (C3) in the form

$$
\begin{gathered}
\hat{\phi}_{R}^{\prime \prime}=\frac{1}{Q} x_{R} \hat{\psi}_{R}^{\prime \prime}, \\
\hat{\psi}_{R}+x_{R} \hat{\phi}_{R}=\frac{1}{Q} \hat{\psi}_{R}^{\prime \prime},
\end{gathered}
$$

where $x_{R}=x / \delta_{L}, \phi_{R}\left(x_{R}\right)=k \delta_{L} \phi\left(\delta_{L} x_{R}\right) /\left(L_{0} \gamma\right)$, and $\psi_{R}\left(x_{R}\right)$ $=\psi\left(\delta_{L} x_{R}\right)$. The parameter $Q$ can be considered as an eigenvalue, while $\delta_{L}$ is an effective layer width, itself implicitly dependent on the eigenvalue. In general, one can assume $\delta_{L} \ll L_{0}$. Thus, there exists an overlapping region $\delta_{L} \ll x$ $\ll L_{0}$ where both Eqs. (C2) and (C3) and the ideal MHD equations (outer equations) are valid. Matching the expressions of the solutions of these two sets of equations in the overlapping region fixes the eigenvalue and the layer width.

The solution of Eqs. (C4) and (C5) was obtained in a closed form, by means of an integral representation, by Coppi et al., ${ }^{82}$ while a detailed calculation is given in the appendix of Ref. 83. An alternative method, ${ }^{84}$ which turns out useful to treat more general models, ${ }^{21,23,85,86}$ is based on Fourier transforms in the variable $x_{R}$.

In the overlapping region, the leading asymptotic expansions of the two functions take the form

$$
\begin{gathered}
\hat{\psi}_{R} \sim \psi_{\infty}\left(1+\frac{\Delta^{\prime}}{2} x\right) \\
\hat{\phi}_{R} \sim \phi_{\infty}\left(1-\frac{2 \lambda_{H}}{\pi} \frac{1}{x}\right),
\end{gathered}
$$

where the quantity $\lambda_{H}$ is a normalised version of the potential energy variation $\delta W$ arising in the energy principle.

The above relations are those pertinent to this work, which is unstable tearing modes $\left(\Delta^{\prime}>0\right)$, but stable ideal modes $\left(\lambda_{H}<0\right)$.

By inspecting Ohm's law (C5) in the overlapping region $x_{R} \gg 1$, one can see that the following relation holds:

$$
1=-\Delta^{\prime} \frac{\lambda_{H}}{\pi}
$$

The eigenvalue condition of Ref. 82 rewritten in terms of ${ }^{23} Q$ and $\Delta^{\prime}$ can be conveniently recast in the form

$$
\Delta^{\prime} \delta_{L}=-\frac{\pi}{8} Q \frac{\Gamma[(Q-1) / 4]}{\Gamma[(Q+5) / 4]} .
$$


From this equation, the scaling of the different $\Delta^{\prime}$ regimes can be immediately recovered. In particular, for $\Delta^{\prime} \delta_{L} \rightarrow 0$, where $\delta_{L} \rightarrow \delta_{T}$, one has to take $Q \rightarrow 0$. In the resistive regime, one takes $d_{e}=0$ so that $\delta_{e}^{2}=\hat{\eta} / \gamma$. One then recovers the scaling (8) for the growth rate and the scaling (9) for the layer width. In the opposite limit, when $\Delta^{\prime} \delta_{L} \rightarrow \infty$ and $\delta_{L} \rightarrow \delta_{K}$, one has to take $Q \rightarrow 1$, to approach the pole of the $\Gamma$ function. The result is then the scaling (10) for the growth rate and the scaling (11) for the layer width. Similarly, in the collisionless regime, one takes $\hat{\eta}=0, \delta_{e}=d_{e}$, and one recovers the collisionless scaling used in the last part of this work.

Finally, we draw the attention to the fact that the scaling of $Q$ can also be estimated directly from Eqs. (C4) and (C5) without carrying out a detailed matching calculation, by taking the constant- $\psi$ scaling $\psi_{R}^{\prime \prime} \sim \Delta^{\prime} \delta_{L} \psi_{R}$ for small $\Delta^{\prime}$ and the natural scaling $\psi_{R}^{\prime \prime} \sim \psi_{R}$ in the large $\Delta^{\prime}$ limit. Then, the condition that all the terms of Eqs. (C4) and (C5) are balanced requires assuming $Q \sim \Delta^{\prime} \delta_{L}$ and $Q \sim 1$ in the two respective regimes. In this way, one makes direct contact with the collisional estimates given in Sec. II.

The corresponding scaling in the collisionless regime is

$$
\begin{gathered}
\gamma_{T} \tau_{0} \sim\left(k d_{e}\right)\left(\Delta^{\prime} d_{e}\right)^{2}, \\
\frac{\delta_{T}}{L_{0}} \sim\left(\frac{d_{e}}{L_{0}}\right)^{2}\left(\Delta^{\prime} L_{0}\right), \\
\gamma_{K} \tau_{0} \sim k d_{e}, \\
\frac{\delta_{K}}{L_{0}} \sim \frac{d_{e}}{L_{0}} .
\end{gathered}
$$

This latter scaling has been used to derive the estimates in Eqs. (34)-(37).

${ }^{1}$ K. Shibata and S. Tanuma, Earth Planets Space 53, 473 (2001).

${ }^{2}$ N. F. Loureiro, S. C. Cowley, W. D. Dorland, M. G. Haines, and A. A. Scheckochihin, Phys. Rev. Lett. 95, 235003 (2005).

${ }^{3}$ N. F. Loureiro, A. A. Scheckochihin, and S. C. Cowley, Phys. Plasmas 14, 100703 (2007).

${ }^{4}$ W. Daughton, V. Roytershteyn, B. J. Albright, H. Karimabadi, L. Yin, and K. J. Bowers, Phys. Rev. Lett. 103, 065004 (2009).

${ }^{5}$ D. Uzdensky, N. F. Loureiro, and A. A. Scheckochihin, Phys. Rev. Lett. 105, 235002 (2010).

${ }^{6}$ F. Pucci and M. Velli, Astrophys. J. Lett. 780, L19 (2014).

${ }^{7}$ A. Tenerani, A. F. Rappazzo, M. Velli, and F. Pucci, Astrophys. J. 801, 145 (2015).

${ }^{8}$ S. Landi, L. Del Zanna, E. Papini, F. Pucci, and M. Velli, Astrophys. J. 806, 131 (2015).

${ }^{9}$ A. Tenerani, M. Velli, A. F. Rappazzo, and F. Pucci, Astrophys. J. Lett. 813, L32 (2015).

${ }^{10}$ D. Del Sarto, F. Pucci, A. Tenerani, and M. Velli, J. Geophys. Res. 121, 1857, doi:10.1002/2015JA021975 (2016).

${ }^{11}$ A. Tenerani, M. Velli, F. Pucci, S. Landi, and A. F. Rappazzo, J. Plasma Phys. 82, 535820501 (2016).

${ }^{12}$ P. A. Cassak and J. F. Drake, Astrophys. J. 707, L158 (2009).

${ }^{13}$ H. Furth, J. Killeen, and M. N. Rosenbluth, Phys. Fluids 6, 459 (1963).

${ }^{14}$ E. R. Priest, Rep. Prog. Phys. 48, 955 (1985).

${ }^{15}$ M. Velli and A. W. Hood, Solar Phys. 119, 107 (1989).

${ }^{16}$ A. Battacharjee, Y.-M. Huang, H. Yang, and B. N. Rogers, Phys. Plasmas 16, 112102 (2009).

${ }^{17}$ E. G. Harris, Il Nuovo Cimento 23, 115 (1962).

${ }^{18}$ S. Von Goeler, W. Stodiek, and N. Sauthoff, Phys. Rev. Lett. 33, 1201 (1974).
${ }^{19}$ A. W. Edwards, D. J. Campbell, W. W. Engelhardt, H.-U. Fahrbach, R. D. Gill, R. S. Granetz, S. Tsuji, B. J. D. Tubbing, A. Weller, J. Wesson, and D. Zasche, Phys. Rev. Lett. 57, 210 (1986).

${ }^{20}$ J. Wesson, Nucl. Fusion 30, 2545 (1990).

${ }^{21}$ F. Porcelli, Phys. Rev. Lett. 66, 425 (1991).

${ }^{22}$ M. Ottaviani and F. Porcelli, Phys. Rev. Lett. 71, 3802 (1993).

${ }^{23}$ M. Ottaviani and F. Porcelli, Phys. Plasmas 2, 4104 (1995).

${ }^{24}$ F. L. Waelbroeck, Phys. Fluids B 1, 2372 (1989).

${ }^{25}$ F. L. Waelbroeck, Phys. Rev. Lett. 70, 3259 (1993).

${ }^{26}$ Q. Yu, S. Günter, and K. Lackner, Nucl. Fusion 54, 072005 (2014).

${ }^{27}$ S. Günter, Q. Yu, K. Lackner, A. Bhattacharjee, and Y. M. Huang, Plasma Phys. Controlled Fusion 57, 014017 (2015).

${ }^{28}$ T. Tajima and K. Shibata, Plasma Astrophysics (Addison-Wesley, 1997), p. 229.

${ }^{29}$ D. Biskamp, Magnetic Reconnection in Plasmas (Cambridge University Press, 2000).

${ }^{30}$ S. V. Bulanov, J. Sakai, and S. I. Syrovatskii, Sov. J. Plasma Phys. 5, 157 (1979).

${ }^{31}$ S. I. Syrovatskii, Rev. Astron. Astrophys. 19, 163 (1981).

${ }^{32}$ D. Del Sarto, F. Califano, and F. Pegoraro, Phys. Rev. Lett. 91, 235001 (2003).

${ }^{33}$ D. Del Sarto, F. Califano, and F. Pegoraro, Mod. Phys. Lett. B 20, 931 (2006).

${ }^{34}$ N. F. Loureiro, A. A. Schekochihin, and D. A. Uzdensky, Phys. Rev. E 87, 013102 (2013).

${ }^{35}$ P. A. Sweet, in Electromagnetic Phenomena in Cosmical Physics, edited by B. Lehnert (IAU Symposium, 1958) Vol. 6, p. 123.

${ }^{36}$ E. N. Parker, J. Geophys. Res. 62, 509-520, doi:10.1029/ JZ062i004p00509 (1957).

${ }^{37}$ J. F. Drake and R. G. Kleva, Phys. Rev. Lett. 66, 1458 (1991).

${ }^{38}$ M. E. Mandt, R. E. Denton, and J. F. Drake, Geophys. Res. Lett. 21, L73, doi:10.1029/93GL03382 (1994).

${ }^{39}$ D. Biskamp, E. Schwarz, and J. F. Drake, Phys. Rev. Lett. 75, 3850 (1995).

${ }^{40}$ M. A. Shay, J. F. Drake, B. N. Rogers, and R. E. Denton, J. Geophys. Res.-Space 106, 3759 (2001).

${ }^{41}$ M. Hesse, J. Birn, and M. Kuznetsova, J. Geophys. Res. 106, 3721, doi:10.1029/1999JA001002 (2001).

${ }^{42}$ J. Birn, J. F. Drake, A. Shay, B. N. Rogers, R. E. Denton, M. Hesse, M. Kuznetsova, Z. W. Ma, A. Battacharjee, A. Otto, and L. Pritchett, J. Geophys. Res. 106, 3715, doi:10.1029/1999JA900449 (2001).

${ }^{43}$ X. Wang and A. Battacharjee, Phys. Rev. Lett. 70, 1627 (1993).

${ }^{44}$ R. G. Kleva, J. F. Drake, and F. L. Waelbroeck, Phys. Plasmas 2, 23 (1995).

${ }^{45}$ R. Fitzpatrick and F. Porcelli, Phys. Plasmas 11, 4713 (2004); 14, 049902 (2007).

${ }^{46}$ J. M. Finn and P. K. Kaw, Phys. Fluids 20, 72 (1977).

${ }^{47}$ P. L. Pritchett and C. C. Wu, Phys. Fluids 22, 2140 (1979).

${ }^{48}$ B. U. O. Sonnerup and J.-I. Sakai, EOS Trans. Am. Geophys. Union 62, 353 (1981).

${ }^{49}$ D. Biskamp, Z. Naturforsch. 37a, 840 (1982).

${ }^{50}$ D. Biskamp, Phys. Lett. 87 A, 357 (1982).

${ }^{51}$ B. Kliem, M. Karlický, and A. O. Benz, Astron. Astrophys. 360, 715 (2000), available at http://aa.springer.de/papers/0360002/2300715/small. htm.

${ }^{52}$ S. I. Syrovatskii, Sov. Phys. JETP 33, 933 (1971), available at http://jetp. ac.ru/cgi-bin/dn/e_033_05_0933.pdf.

${ }^{53}$ B. D. Jemella, M. A. Shay, J. F. Drake, and B. N. Rogers, Phys. Rev. Lett. 91, 125002 (2003).

${ }^{54}$ D. Del Sarto, F. Califano, and F. Pegoraro, Phys. Plasmas 12, 012317 (2005).

${ }^{55}$ J. F. Drake, R. G. Kleva, and M. E. Mandt, Phys. Rev. Lett. 73, 1251 (1994).

${ }^{56}$ J. F. Drake, M. Swisdak, K. M. Schoeffler, B. N. Rogers, and S. Kobayashi, Geophys. Res. Lett. 33, L13105, doi:10.1029/2006GL025957 (2006).

${ }^{57}$ W. Daughton, J. Scudder, and H. Karimabadi, Phys. Plasmas 13, 072101 (2006).

${ }^{58}$ A. Y. Aydemir, Phys. Fluids B 4, 3469 (1992).

${ }^{59}$ S. Tanuma, T. Yokoyama, T. Kudoh, and K. Shibata, Astrophys. J. 551, 312 (2001).

${ }^{60}$ K. Shibata, Adv. Space Res. 17, 9 (1996).

${ }^{61}$ R. Samtaney, N. F. Loureiro, D. A. Uzdensky, A. A. Schekochihin, and S. C. Cowley, Phys. Rev. Lett. 103, 105004 (2009). 
${ }^{62}$ J. Lin, S. R. Cranmer, and C. J. Farrugia, J. Geophys. Res. 113, A11107, doi:10.1029/2008JA013409 (2008).

${ }^{63}$ G. Lapenta, Phys. Rev. Lett. 100, 235001 (2008).

${ }^{64}$ A. Biancalani and B. D. Scott, Europhys. Lett. 97, 15005 (2012).

${ }^{65}$ L. Comisso, D. Grasso, F. L. Waelbroeck, and D. Borgogno, Phys. Plasmas 20, 092118 (2013).

${ }^{66}$ A. Ali, J. Li, and Y. Kishimoto, Phys. Plasmas 21, 052312 (2014).

${ }^{67}$ P. A. Cassak, R. N. Taylor, R. L. Fermo, M. T. Beidler, M. A. Shay, M. Swidsak, J. F. Drake, and H. Karimabadi, Phys. Plasmas 22, 020705 (2015).

${ }^{68}$ M. Hirota, Y. Hattori, and P. J. Morrison, Phys. Plasmas 22, 052114 (2015).

${ }^{69}$ D. A. Uzdensky and N. F. Loureiro, Phys. Rev. Lett. 116, 105003 (2016).

${ }^{70}$ L. Comisso, M. Lingham, Y. M. Huang, and A. Battacharjee, Phys. Plasmas 23, 100702 (2016).

${ }^{71}$ M. Wan, A. F. Rappazzo, W. H. Matthaeus, S. Servidio, and S. Oughton, Astrophys. J. 797, 63 (2014).

${ }^{72}$ J. P. Eastwood, T.-D. Phan, F. S. Mozer, M. A. Shay, M. Fujimoto, A. Retinò, M. Hesse, A. Balogh, E. A. Lucek, and I. Dandouras, J. Geophys. Res. 112, A6235, doi:10.1029/2006JA012158 (2007).

${ }^{73}$ R. Wang, Q. Ly, A. Du, and S. Wang, Phys. Res. Lett. 104, 175003 (2010).

${ }^{74}$ A. T. Lin, Phys. Fluids 21, 1026 (1978).
${ }^{75}$ Y. Ishi, M. Azumi, and Y. Kishimoto, Phys. Rev. Lett. 89, 205002 (2002).

${ }^{76}$ Z. X. Wang, X. G. Wang, J. Q. Dong, Y. A. Lei, Y. X. Long, Z. Z. Mou, and W. X. Qu, Phys. Rev. Lett. 99, 185004 (2007).

${ }^{77}$ M. Janvier, Y. Kishimoto, and J. Q. Li, Phys. Rev. Lett. 107, 195001 (2011).

${ }^{78}$ J. Jara-Almonte, H. Ji, M. Yamada, J. Yoo, and W. Fox, Phys. Rev. Lett. 117, 095001 (2016).

${ }^{79}$ X. Xu, J. Wang, Y. Wen, Y. Yu. A. Liu, T. Lan, C. Yu, B. Wan, X. Gao, Y. Sun, N. C. Luhmann, Jr., C. W. Domier, Z. G. Xia, and Z. Shen, Plasma Phys. Controlled Fusion 52, 015008 (2010).

${ }^{80}$ V. Igochine, J. Boom, I. Classen, O. Dumbrajs, S. Günter, K. Lackner, G. Pereverzev, H. Zohm, and ASDEX Upgrade Team, Phys. Plasmas 17, 122506 (2010).

${ }^{81}$ A. L. Moser and P. M. Bellan, Nature 482, 379 (2012).

${ }^{82}$ B. Coppi, R. Galvão, R. Pellat, M. Rosenbluth, and P. Rutherford, Fiz. Plazmy 2, 961 (1976).

${ }^{83}$ G. Ara, B. Basu, and B. Coppi, Ann. Phys. 112, 443 (1978).

${ }^{84}$ F. Pegoraro and T. J. Schep, Plasma Phys. Controlled Fusion 4, 667 (1986).

${ }^{85}$ F. Pegoraro, F. Porcelli, and T. J. Schep, Phys. Fluids B 1, 364 (1989).

${ }^{86}$ S. V. Bulanov, F. Pegoraro, and A. S. Sakharov, Phys. Fluids B 28, 647 (1992). 